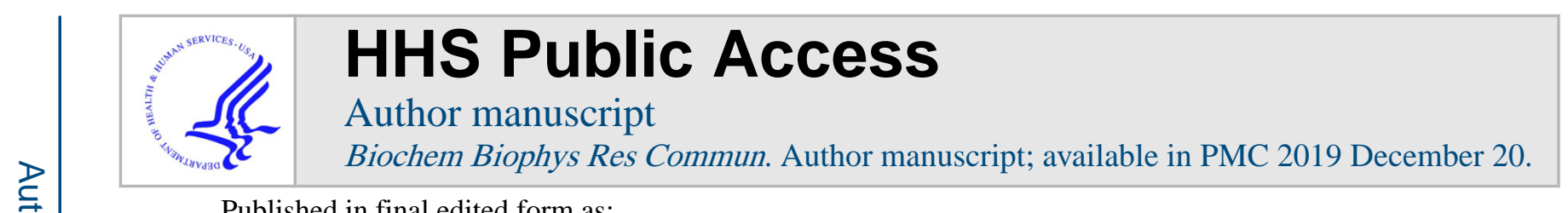

Published in final edited form as:

Biochem Biophys Res Commun. 2018 October 07; 504(3): iii. doi:10.1016/j.bbrc.2018.09.117.

\title{
Editorial for BBRC lipidomics special issue
}

\author{
Sarah Spiegel, James Ntambi
}

The ability to analyze large numbers of lipids and metabolites has greatly advanced the understanding of their biochemical functions and roles in disease, but has also created challenges of scale. This mini review series covers areas of bioactive lipid metabolism and research that have been particularly impacted by these new technologies, and their potential to transform precision medicine. Metabololipidomics provides a system based technology to interrogate the bioactive mediators, their pathways and further metabolites involved in animal and human physiology and pathophysiology. In this elegant review, Norris and Serhan [1] describe novel metabololipidomic approaches using mass spectrometry for the identification and profiling of proresolving lipid mediators that control inflammation, particularly in blood coagulation and in solid tumors. Saeki et al. [2] described a LC-MS/MS improved multiplex method to simultaneously profile almost 100 bioactive lipids in different dendritic cell subsets. This timely review by Wood et al. [3] is on the limitations of the use of high resolution lipidomics for identification of biomarkers and how biomarkers should be validated by targeted, quantitative assays. Future research needs to address these topics as they are essential for the translation of biomarker research to clinical practice. In this context, Lee and colleagues [4] in their review also summarized current limitations and challenges of LC-ESI-MS/MS targeted lipidomics and non-targeted lipidomics.

The tumor microenvironment is now receiving much attention for its roles in regulating tumor progression and metastasis. Patterson et al. [5] highlighted the importance of abnormal lipid metabolism in tumors and their microenvironment in tumor progression, especially in the less well understood process of lipophagy due to aberrant degradation of lipids via autophagy. The review by Miyazaki et al. [6] is an update on lipidomics analysis related to cardiovascular diseases conditions that are generally caused by blocked blood vessels that can lead to a heart attack. There is a special emphasis in this review on the identification of biomarkers to potentially predict cardiovascular events in younger patients. Herr et al. [7] first discussed how advances in high throughput mass spectrometry have led to detailed lipidomic profiles in large cohorts of clinical samples. In part 2, these authors [8] discussed the potential impact of sphingolipidomics from large cohorts of patients on patient care and diagnosis. The review by Cowart and colleagues [9] discusses the use of targeted sphingolipidomics in non-alcoholic fatty liver disease in animal models and human patient samples. While most data thus far are derived from targeted lipidomics approaches, unbiased, semi-quantitative approaches hold additional promise for furthering our understanding of sphingolipids as biomarkers of and players in non-alcoholic fatty liver disease, that is now emerging as a major clinical concern. Griffiths et al. [10] reviewed targeted lipidomic approaches that have been used to make some recent discoveries in the biochemistry of oxysterols including regulation of innate and adaptive immune systems and their roles in tumor suppression. The review by Fuller and Futerman [11] is focused on dyshomeostasis of membrane lipids in neurodegenerative lysosomal storage disorders, and 
discusses perturbations of lipid metabolism and effects on neuronal membranes that impair their functions.

\section{References}

[1]. Norris Paul C., Serhan Charles N., Metabololipidomic profiling of functional immunoresolvent clusters and eicosanoids in mammalian tissues, Biochem. Biophys. Res. Commun 504 (2018) 553-561. [PubMed: 29524409]

[2]. Ohba Mai, Saeki Kazuko, Koga Tomoaki, Okuno Toshiaki, Kobayashi Yuichi, Yokomizo Takehiko, Profiling of bioactive lipids in different dendritic cell subsets using an improved multiplex quantitative LC-MS/MS method, Biochem. Biophys. Res. Commun 504 (2018) $562-$ 568. [PubMed: 29890138]

[3]. Wood Paul L., Cebak John E., Lipidomics biomarker studies: errors, limitations, and the future, Biochem. Biophys. Res. Commun 504 (2018) 569-575. [PubMed: 29596837]

[4]. Lee Hyeon-Cheol, Yokomizo Takehiko, Applications of mass spectrometry-based targeted and non-targeted lipidomics, Biochem. Biophys. Res. Commun 504 (2018) 576-581. [PubMed: 29534960]

[5]. Maan Meenu, Peters Jeffrey M., Dutta Mainak, Patterson Andrew D., Lipid metabolism and lipophagy in cancer, Biochem. Biophys. Res. Commun 504 (2018) 582-589. [PubMed: 29438712]

[6]. Kohno Shohei, Keenan Audrey L., Ntambi James M., Miyazaki Makoto, Lipidomic insight into cardiovascular diseases, Biochem. Biophys. Res. Commun 504 (2018) 590-595. [PubMed: 29665359]

[7]. Chew Wee Siong, Seow Wei Lun, Chong Joyce R., Lai Mitchell K.P., Torta Federico, Wenk Markus R., Herr Deron R., Sphingolipidomics analysis of large clinical cohorts. Part 1: technical notes and practical considerations, Biochem. Biophys. Res. Commun 504 (2018) 596-601. [PubMed: 29654754]

[8]. Chong Joyce R., Xiang Ping, Wang Wei, Hind Tatsuma, Chew Wee Siong, Ong Wei-Yi, Lai Mitchell K.P., Herr Deron R., Sphingolipidomics analysis of large clinical cohorts. Part 2: potential impact and applications, Biochem. Biophys. Res. Commun 504 (2018) 602-607. [PubMed: 29654757]

[9]. Montefusco David J., Allegood Jeremy C., Spiegel Sarah, Cowart L Ashley, Non-alcoholic fatty liver disease: insights from sphingolipidomics, Biochem. Biophys. Res. Commun 504 (2018) 608-616. [PubMed: 29778532]

[10]. Griffiths William J., Wang Yuqin, An update on oxysterol biochemistry: new discoveries in lipidomics, Biochem. Biophys. Res. Commun 504 (2018) 617-622. [PubMed: 29421651]

[11]. Fuller Maria, Futerman Anthony H., The brain lipidome in neurodegenerative lysosomal storage disorders, Biochem. Biophys. Res. Commun 504 (2018) 623-628. [PubMed: 29524416] 